\title{
Post-COVID implications for physician wellness, education, and clinical medicine
}

\author{
Kiran Grant ${ }^{1} \oplus \cdot$ Shaun Mehta $^{2} \cdot$ Alun Ackery $^{3}$
}

Received: 1 March 2021 / Accepted: 11 May 2021 / Published online: 19 June 2021

() The Author(s), under exclusive licence to Canadian Association of Emergency Physicians (CAEP)/ Association Canadienne de Médecine d'Urgence (ACMU) 2021

Keywords COVID-19 $\cdot$ Wellness $\cdot$ Burnout $\cdot$ Education $\cdot$ Virtual care

Dear Editor,

The COVID-19 pandemic will have a longstanding impact on all levels of business, government and healthcare. While healthcare workers are actively engaged on the frontlines, the healthcare system they inhabit is rapidly changing around them. The health and wellness of providers have been further stressed, increased virtual care utilization is changing healthcare delivery, and medical education and research dissemination has rapidly shifted to virtual platforms.

Maintaining mental and physical health has been a welldocumented challenge during this global pandemic for all healthcare workers [1]. While serving on the front lines has increased clinical demands on emergency physicians, their lives outside the department are also more stressful due to widespread economic hardship, fear of infecting family members, ever-changing childcare and family responsibilities, and potential loss of traditional coping mechanisms [2]. To address these challenges, emergency departments (ED) should consider recruiting additional allied healthcare and administrative staff, including medical assistants, scribes, coordinators, and clerical assistants to unburden emergency medicine (EM) physicians from nonclinical tasks. Mental health supports, offered both digitally and in-person, and scheduling flexibility, could also provide additional support for staff in times of constant distress.

Kiran Grant

kiran.grant@mail.utoronto.ca

1 Faculty of Medicine, University of Toronto, Toronto, ON, Canada

2 Emergency Department, St. Michael's Hospital, Unity Health Toronto, Toronto, ON, Canada

3 St. Michael's Hospital, Unity Health Toronto, Toronto, ON, Canada
As other physicians bring virtual care options into their practice healthcare will be more accessible, and there may be a reduced number of patients presenting to the ED for non-emergent health issues. EM physicians may themselves start providing virtual care to further reduce preventable ED visits, and virtual care could be leveraged to provide followup for ED patients, improving care accessibility and reducing return visits [3]. To realize these benefits, the patient presentations appropriate for virtual care need to be clearly defined, best practices on provider training from other systems in the UK and US should be explored, all while remaining mindful of the "digital inequity" that virtual care could perpetuate [4].

Although the shift to virtual platforms inherently has drawbacks in medical education and research dissemination, these circumstances also enable greater collaboration between physically disparate clinicians and researchers and could inspire the creation of even higher fidelity simulation that could greatly advance medical education [5].

With this Pandemic, despite its hardships, there may be an unseen benefit. Frontline staff has a unique opportunity to learn from the challenges encountered during this time and lead emergency medicine to places not imagined previously. Proactive and comprehensive initiatives to promote wellness, utilizing virtual care, and technological enabled collaboration in medical education and research could not only benefit EM during COVID-19 but also serve as a springboard to a brighter post-COVID future.

Conflict of Interest None of the three authors have any conflicts of interest to disclose. 


\section{References}

1. Lim R, Aarsen KV, Gray S, Rang L, Fitzpatrick J, Fischer L. Emergency medicine physician burnout and wellness in Canada before COVID19: a national survey. Can J Emerg Med. 2020;22(5):603-7.

2. Lai J, Ma S, Wang Y, Cai Z, Hu J, Wei N, et al. Factors associated with mental health outcomes among health care workers exposed to Coronavirus disease 2019. JAMA Netw Open. 2020;3(3):e203976-e203976.
3. Dorsey ER, Topol EJ. State of telehealth. N Engl J Med. 2016;375(2):154-61.

4. Achenbach SJ. Telemedicine: benefits challenges, and its great potential. Health Law Policy Brief. 2020;1:26.

5. Bracq M-S, Michinov E, Jannin P. Virtual reality simulation in nontechnical skills training for healthcare professionals: a systematic review. Simul Healthc. 2019;14(3):188-94. 